\section{APP-MAC-PHY Cross-Layer Video Streaming Technique over Wireless Channels}

\author{
Jaeyoung Park', Jaekwon Kim
}

\section{ABSTRACT}

In this letter, we propose a cross-layer technique jointly considering modulation coding schemes (MCSs) of medium access control (MAC) layer, source significance information (SSI) and error concealment unit of application (APP) layer, and channel quality information (CQI) of physical (PHY) layer. We demonstrate the improved video quality by the proposed technique when H.264 videos are streamed over Rayleigh fading wireless channels.

Key Words : Cross-layer technique, Unequal error protection (UEP), Wireless video, Video streaming, Modulation coding schemes (MCS)

\section{I . Introduction}

Error-resilient video streaming under wireless channel environments is a challenging task mainly due to unstable channels ${ }^{[1]}$. In order to overcome the unstable channel environments, unequal error protection (UEP) techniques can be used ${ }^{[2]}$. The multi-antenna techniques of WirelessMANAdvanced systems were used to implement the concept of $\mathrm{UEP}^{[3]}$.

In this paper, we propose a novel cross-layer technique to implement UEP for robust video streaming over wireless channels. We assume Rayleigh flat fading channels between a single transmit and a single receive antenna, 10 MCS levels in MAC layer, and H.264/AVC videos in
Table 1. 10 MCS levels used in the simulations.

\begin{tabular}{c|c|c|c|c}
\hline $\begin{array}{c}\text { MCS } \\
\text { level }\end{array}$ & modulation & $\begin{array}{c}\text { code } \\
\text { rate }\end{array}$ & repetition & puncturing \\
\hline 1 & QPSK & $1 / 12$ & 6 & {$[111111111111]$} \\
\hline 2 & QPSK & $1 / 6$ & 3 & {$[11111111111]$} \\
\hline 3 & QPSK & $1 / 3$ & 2 & {$[110110111110]$} \\
\hline 4 & QPSK & $1 / 2$ & 1 & {$[11111111111]$} \\
\hline 5 & QPSK & $2 / 3$ & 1 & {$[110110111110]$} \\
\hline 6 & 16-QAM & $1 / 2$ & 1 & {$[11111111111]$} \\
\hline 7 & 16-QAM & $2 / 3$ & 1 & {$[110110111110]$} \\
\hline 8 & 16-QAM & $2 / 4$ & 1 & {$[110110011011]$} \\
\hline 9 & 64-QAM & $2 / 3$ & 1 & {$[110110111110]$} \\
\hline 10 & 64-QAM & $5 / 6$ & 1 & {$[1101100110]$} \\
\hline
\end{tabular}

APP layer. The proposed technique imporves the video quality in two steps. In the first step, the video quality is improved by deciding the threshold channel signal-to-noise ratios (SNRs) of $10 \mathrm{MCS}$ considering the error concealment unit of APP layer. In the second step, the video quality is improved by selecting MCS level considering SSI as well as CQI. We demonstrate that the proposed APP-MAC-PHY cross-layer technique offers improved video quality. The improved performance comes at the cost of slightly reduced data transmission speed.

\section{Conventional Systems}

Physical Layer : The Rayleigh flat fading channel is realized by the Jakes' model. The product of symbol time $T_{s}$ and Doppler frequency $f_{D}$ is $6.7 \mu$. A PHY packet is composed of 600 symbols. The adopted channel coding scheme is the convolutional coding with constraint length 9 with polynomials of [110101111] and [100011101]. Soft Viterbi decoding and unquantized soft value at the receiver side are assumed.

Medium Access Control Layer : We consider 10 MCS levels in Table I, which is the same as the IEEE 802.16e standard. The packet error rates (PERs) of 10 MCS levels in the AWGN channel are given in Fig. 1. The PERs can be expressed as PER

※ This research was supported by Global PH.D Fellowship Program through the National Research Foundation of Korea(NRF) funded by the Ministry of Education (NRF-2013H1A2A1032531).

- First Author : Computer and Telecommun. Engineering, Yonsei University, Wonju, rtyua@hanmail.net, 학생회원

Corresponding Author : Computer and Telecommun. Engineering, Yonsei University, Wonju, jaekwon@yonsei.ac.kr, 종신회원 논문번호 : KICS2014-06-244, Received June 25, 2014; Revised July 7, 2014; Accepted July 7, 2014 


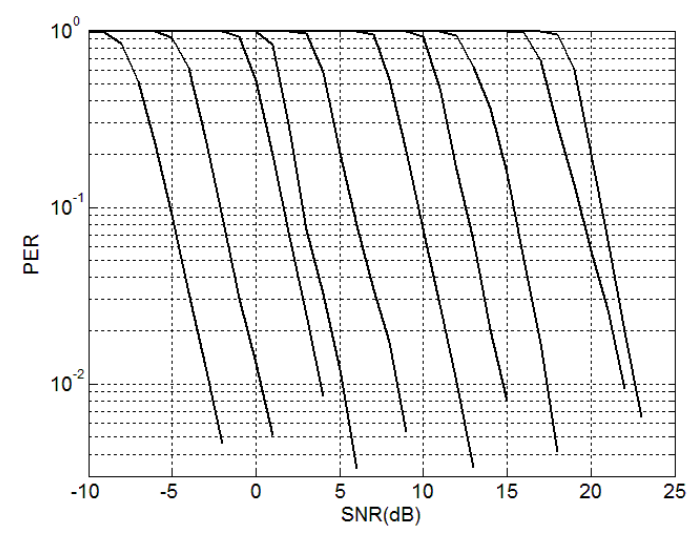

Fig. 1. Packet error rate performance of 10 MCS levels in Table 1

${ }_{L}=f_{L}(S N R)$, where $f_{L}(\cdot)$ is the PER-SNR function of the $L$ th MCS level. When a target PER PER $\mathrm{T}_{\mathrm{T}}$ is given, the threshold SNRs can be calculated for each MCS levels as follows.

$$
\mathrm{SNR}_{\mathrm{TH}, L}=f_{L}^{-1}\left(\mathrm{PER}_{\mathrm{T}}\right), L=1,2, \cdots 10
$$

In the MAC layer, the MCS level $L$ is selected for the following channel condition.

$$
\mathrm{SNR}_{\mathrm{TH}, L} \leq \frac{\left|h_{k}\right|^{2}}{\sigma_{z}^{2}}<\mathrm{SNR}_{\mathrm{TH}, L+1}
$$

where $h_{k}$ denotes the channel gain during the $k$ th PHY packet.

Application Layer: Considered video compression technique is H.264/AVC that is known to be commercially successful. A GOP is composed of 1 I-picture and 15 P-pictures without B-pictures. Thus source significance information is easy to calculate. When a video sequence is divided into 4 consecutive equal length segments, the first segment is the most important and the last segment is the least important, and so on.

\section{Proposed Cross-Layer Technique}

In the conventional system in Section II, the 3 layers perform separately resulting in insufficient video quality. We improve the video quality in two steps.

Step 1: We improve the video quality by incorporating the error concealment unit of APP layer in the calculation of threshold SNRs of MCS levels. The target PER for each MCS level is set as follows.

$$
\operatorname{PER}_{\mathrm{T}, L}=\operatorname{PER}_{\mathrm{T}, 10} \frac{\operatorname{size}_{L}}{\operatorname{size}_{10}}, L=1,2, \cdots, 10 .
$$

where size ${ }_{L}$ denotes the PHY packet size in bits of MCS level $L$. Then the threshold SNRs for MCS levels are decided as follows.

$$
\mathrm{SNR}_{\mathrm{TH}, L}=f_{L}^{-1}\left(\operatorname{PER}_{\mathrm{T}, L}\right), L=1,2, \cdots, 10 .
$$

The above PERs in (3) and their corresponding threshold SNRs in (4) guarantee that the probability of error of the APP layer data unit such as I-picture (or P-picture) are the same regardless of the selected MCS level. Since a PHY packet is composed of 600 symbols, the payload data for the MCS level 1 and 10 are 100 bits and 3000bits, respectively. The different amount of payload data of MCS levels means that an APP layer data unit is translated into different number of PHY packets. Note that when error concealment method for video is frame(picture)-copy, the error of a single PHY packet is the same as the error of the whole picture. If an APP layer error concealment unit is composed of 100 PHY packets for MCS level $L$ and 200 PHY packets for MCS level $L^{\prime}$, the target PER of MCS level $L^{\prime}$ needs to be a half of that of level $L$ for the same video quality.

Step 2 : For the H.264/AVC videos described in Section II, 4 video segments have 4 different source significance information $S=1,2,3,4$. Obviously the first segment (that contains the I-picture) with $S=1$ is of the highest importance in terms of PSNR performance. In an effort to improve the video quality, we incorporate SSI information $S$ in deciding MCS level $L$ as follows. 


$$
\mathrm{SNR}_{\mathrm{TH}, L} \leq \frac{\left|h_{k}\right|^{2}}{\sigma_{z}^{2}}+\operatorname{offset}_{S}<\operatorname{SNR}_{\mathrm{TH}, L+1}
$$

where offset ${ }_{S}$ is given as $-\Delta / 2,-\Delta / 4, \Delta / 4, \Delta / 2$ for $S=1,2,3,4$, respectively, and $\Delta$ is calculated as follows.

$$
\Delta=\sum_{L=10}^{10} \mathrm{SNR}_{\mathrm{TH}, L} / 9
$$

We transmit more important data slower and less important data faster than the conventional streaming method, thereby implementing the concept of unequal error protection.

\section{Simulation}

In this section, we perform computer simulations to show the improved video quality by the proposed technique. The adopted video is $\mathbf{2 5 6}$ frame Foreman of CIF resolution. We transmitted the Foreman sequence 10 times and averaged the 10 PSNR values. Fig. 2 shows the average PSNR performance of the two streaming methods when the average channel SNR is $15 \mathrm{~dB}$; the conventional streaming method using (1) and (2) and the proposed method using (4) and (5). We can observe the improvement of video quality by the proposed two Steps in Section III. As can be seen in Fig. 2, the PSNRs of the conventional streaming method is significantly improved by the proposed technique especially for the last two GOPs (frame number 224 256). Table 2 compares the average PSNR performance for different channel SNR values, demonstrating the improved performance for various channel SNR values.

\section{Conclusion}

In this letter, we proposed a cross-layer video streaming technique considering APP layer, MAC layer, and PHY layer together. The proposed method uses the error concealment data unit, source significance information, and channel quality

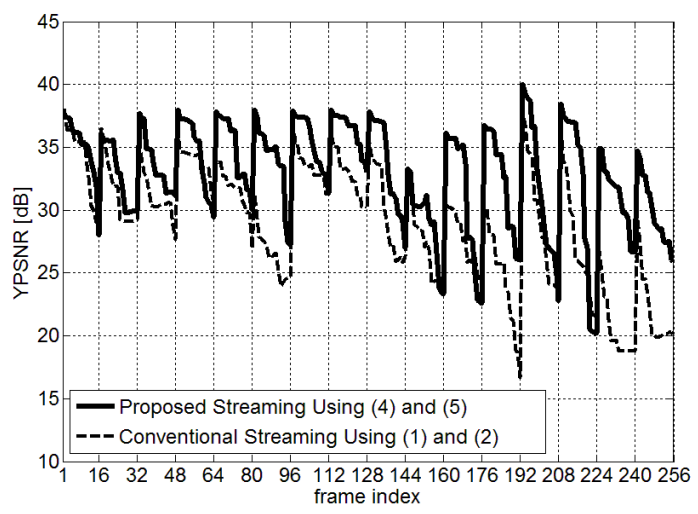

Fig. 2. Comparison of the conventional video streaming method and the proposed streaming method in terms of PSNR performance when channel SNR is $15[\mathrm{~dB}]$.

Table 2. Average PSNRs[dB] of the coventional video streaming method and the proposed streaming method for various channel SNR values.

\begin{tabular}{c|c|c|c}
\hline Channel SNR[dB] & 14 & 15 & 16 \\
\hline \hline PSNR by Proposed Tech. [dB] & 32.7 & 32.9 & 33.0 \\
\hline PSNR by Conv. Tech. [dB] & 29.4 & 28.9 & 29.9 \\
\hline
\end{tabular}

information simultaneously. The improved video quality by the proposed method was demonstrated under time varying Rayleigh fading channel environment.

\section{References}

[1] T. Stockhammer, M. M. Hannuksela, and T. Wiegand, "H.264/AVC in wireless environments," IEEE Trans. Circuits Syst. Video Technol., vol. 13, no. 7, pp. 657-673, Jul. 2003.

[2] K. Park, M. Nam, J. Park, and H. Song, "Unequal error protection: survey and standardization prospect," J. KICS, vol. 37, no. 11, pp. 1054-1063, Nov. 2012.

[3] J. Yun, Y. Kim, and J. Kim, "Maximum likelihood UEP video streaming technique for WirelessMAN-Advanced systems" IEEE Trans. Consumer Electronics, vol. 59, no. 1, pp. 175-181, Feb. 2013. 